\title{
Dos nuevas especies colombianas de la sección Brevispicae del género Sloanea (Elaeocarpaceae)
}

\author{
Leonardo Palacios-Duque ${ }^{1 *} \&$ José Luis Fernández-Alonso ${ }^{2}$ \\ ${ }^{1}$ Universidad Nacional de Colombia, Instituto de Ciencias Naturales, apartado 7495 Bogotá D. C., Colombia. lepduque@hotmail.com \\ ${ }^{2}$ Real Jardín Botánico, CSIC, Plaza de Murillo 2, E-28014 Madrid, España. jlfernandeza@rjb.csic.es
}

\section{Resumen}

Palacios-Duque, L. \& J.L. Fernández-Alonso. 2011. Dos nuevas especies colombianas de la sección Brevispicae del género Sloanea (Elaeocarpaceae) . Anales Jard. Bot. Madrid 69(1): 91-95.

Se describen e ilustran Sloanea helianthoides y Sloanea huilaeana como nuevas especies colombianas pertenecientes a la sección Brevispicae del género. Sloanea helianthoides se caracteriza por sus flores con sépalos grandes (7-9 mm de longitud), frutos grandes (de c. $6 \mathrm{~cm}$ de longitud) con espinas agrupadas y con numerosas semillas (más de 20) y Sloanea huilaeana se distingue por sus hojas con venación craspedódroma e indumento cobrizo y por sus espinas del fruto ligeramente curvas que se vuelven más delgadas hacia el ápice de la cápsula. Se ofrece una clave para las especies colombianas de la sección Brevispicae.

Palabras clave: Brevispicae, Colombia, Corymbo-racemi, Elaeocarpaceae, Paniculi, Sloanea.

\section{INTRODUCCIÓN}

Sloanea L., es un género de la familia Elaeocarpaceae que cuenta con unas 150-160 especies distribuidas en las regiones tropicales y subtropicales del mundo, a excepción de África continental (D.A. Smith, 2001, 2011; Sampaio \& Souza, 2011). Las especies de Sloanea se reconocen generalmente por ser árboles que presentan raíces tabulares muy llamativas, con flores generalmente apétalas (en 98 de las 101 especies que crecen en el Neotrópico) y por sus frutos provistos de espinas rígidas o flexibles, algunas veces irritantes (D.A. Smith y Steyermark, 1998). El género se encuentra dividido en dos subgéneros (Quadrisepala Earle Sm. y Sloanea) y en cuatro secciones (Brevispicae Earle Sm., Corymbo-racemi Earle Sm., Paniculi Earle Sm. y Sloanea), categorías infragenéricas adecuadamente descritas en trabajos como los de C.E. Smith (1954), Alfaro (1981) y Vásquez (2009).

La sección neotropical Brevispicae del género Sloanea cuenta con 38 especies, de las que 14 habitan en Colombia. Las especies miembros de esta sección se caracterizan por tener estípulas prontamente caducas, pequeñas, deltoides o lanceoladas; inflorescencias axilares, racemosas; flores con 4 a 12 sépalos desiguales en forma y tamaño, los cuales nunca cubren los estambres en el botón; cápsulas generalmente de 2,5 a 4,5(6) $\mathrm{cm}$ de longitud; espinas cortas o largas, generalmente flexibles e irritantes (C.E. Smith, 1954). Tradicionalmente se consideraba que el género Sloanea en el neo-

\section{Abstract}

Palacios-Duque, L. \& J.L. Fernández-Alonso. 2011. Two new Colombian species of the section Brevispicae of the genus Sloanea (Elaeocarpaceae). Anales Jard. Bot. Madrid 69(1): 91-95 (in spanish).

Two new species from Colombia assigned to Sloanea section Brevispicae, Sloanea helianthoides and S. huilaeana, are described and illustrated. Sloanea helianthoides is characterized by its flowers with large sepaIs (7-9 mm long) and large fruit (c. $6 \mathrm{~cm}$ long) with grouped spines and many seeds (more than 20) and Sloanea huilaeana is distinguished by its craspedodrome venation, coppery indumentum on the leaves, and the fruit spines slightly curved and getting thinner towards the capsule apex. A key to the Colombian species of section Brevispicae is included.

Key words: Brevispicae, Colombia, Corymbo-racemi, Elaeocarpaceae, Paniculi, Sloanea.

trópico sólo presentaba cápsulas con 1-4 semillas, pero el estudio de las colecciones recientes, depositadas en diferentes herbarios de Colombia, ha permitido identificar y describir algunas especies con más de 10 semillas por cápsula, Palacios-Duque (2007). Continuando con el trabajo de revisión de este género para Colombia, en esta entrega, presentamos dos nuevas especies que también poseen cápsulas con abundantes semillas, las cuales son comparadas con las especies consideradas más afines.

\section{MATERIALES Y MÉTODOS}

Estudio morfológico de los especímenes. Se revisaron 73 ejemplares de la sección aquí estudiada (Brevispicae), los cuales se encuentran depositados en los herbarios CAUP, CHOCO, COAH, COL, CUVC, FMB, HUA, HUQ, JAUM, MEDEL, TULV y UDBC. Las técnicas utilizadas en el tratamiento de las colecciones corresponden a las empleadas en los estudios taxonómicos clásicos y la terminología utilizada en la descripción de las estructuras sigue básicamente a Font Quer (1993). Para la delimitación de las categorías infragenéricas y para la identificación de las especies, se tuvieron en cuenta las revisiones y tratamientos clásicos (D.A. Smith, 1954; Alfaro, 1981; Steyermark, 1988; D.A. Smith \& Steyermark, 1998; D.A. Smith, 2010). En el estudio de las descripciones originales y tipos nomenclaturales, se utilizaron también los diferentes recursos electrónicos disponibles en los sitios de internet de los herbarios F, KEW, MO, NY, US. 
El estado de conservación de las especies fue determinado de acuerdo con las categorías de riesgo o amenaza de la UICN, tal como fueron preparadas por la comisión de supervivencia de especies (SSC) IUCN (2001) y con el complemento para la categorización a nivel regional, publicado en el folleto sobre directrices para emplear los criterios de la lista roja de la UICN a nivel nacional y regional en Colombia (IUCN, 2003).

\section{RESULTADOS Y DISCUSIÓN}

Se presenta la descripción de dos nuevos táxones del género Sloanea y una clave para la identificación de las 14 especies hasta ahora conocidas de la sección en Colombia, incluidas las descritas en este trabajo.

\section{CLAVE DE LAS ESPECIES DE LA SECCIÓN BREVISPICAE}

1. Láminas con nervios secundarios rectos hasta el margen, éste con diminutos dientes (originados a partir de los nervios secundarios) en el tercio apical .................................................................... 2

1. Láminas con nervios secundarios arqueados antes de ó en el margen, éste sin dientes diminutos en el tercio apical ...................... 3

2. Cápsulas con una a dos semillas, valvas provistas de espinas de color verde, rectas, rígidas ...... 12. S. pseudogranulosa Pal.-Duque

2. Cápsulas con más de quince semillas, valvas provistas de espinas de color café, curvas, flexibles ......... 8. Sloanea huilaeana, sp. nov.

3. Cápsulas con más de 2 semillas .............................................. 4

3. Cápsulas con una (raramente 2) semillas ........................................ 5

4. Cápsulas elípticas, de $26-29 \mathrm{~mm}$ de longitud, con espinas moradas a negruzcas, esparcidas (separadas 1-2 mm entre sí), fácilmente desprendibles ............................ 5. S. esmeraldana Pal.-Duque

4. Cápsulas ovoides a redondeadas, hasta $60 \mathrm{~mm}$ de longitud, espinas amarillentas, agrupadas (densamente dispuestas, a menos de 1 $\mathrm{mm}$ de distancia), difícilmente desprendibles

7. Sloanea helianthoides, sp. nov.

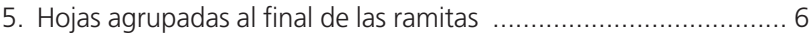

5. Hojas esparcidas sobre las ramitas .......................................... 7

6. Estambres con conectivo delgado y arista estaminal larga, filiforme, de 0,8-2 mm de longitud; cápsula con espinas de 3-5 mm de longitud, pubescencia densa, amarillenta a dorada

13. S. rufa Planch. ex Benth.

6. Estambres con conectivo ancho y arista estaminal corta, gruesa, menor de 0,6 mm de longitud; cápsula con espinas de 7-10 mm de longitud, pubescencia fina y esparcida, café ...... 1. S. brevipes Benth.

7. Sépalos 4; láminas, en seco, con haz y envés del mismo color (verdoso)

14. S. stipitata Spruce ex Benth.

7. Sépalos 5-9; láminas, en seco, con coloración contrastante en haz y envés (discoloras) o uniforme

8. Láminas foliares discoloras, haz café brillante, envés glauco, diminutamente pubérulo

4. S. eichleri K. Schum.

8. Láminas foliares de un solo color, generalmente café o verdoso ....

9. Ramas y ramitas con abundantes yemas; pecíolo con superficie muy estriada y escariosa, que se desprende en escamas

3. S. chocoana Pal -Duque

9. Ramas y ramitas carentes de yemas; pecíolo sin esta característica

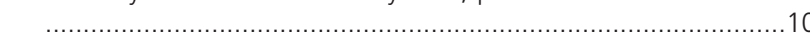

10. Espinas de las cápsulas laxamente dispuestas, separadas $2-3 \mathrm{~mm}$ unas de otras; espinas de 9-12 mm de longitud

11. S. picapica Standley

10. Espinas de las cápsulas densamente agrupadas (dispuestas a menos de $1 \mathrm{~mm}$ unas de otras), espinas de generalmente de menos de $9 \mathrm{~mm}$ de longitud

11. Cápsulas con espinas de 1-3 mm de longitud, superficie de los pecíolos estriada ................................................................ 12

11. Cápsulas con espinas de 3,5-9 mm de longitud, superficie de los pecíolos lisa o estriada
12. Pecíolos de 25-90 mm de longitud, láminas ovado-oblongas; cápsulas de 20-35 mm de longitud; espinas de 2,5-3 mm de longitud, claviformes, amarillentas o cafés .... 2. S. brevispina C.E. Smith Jr.

12. Pecíolos de 10-27 mm de longitud, láminas espatuladas; cápsulas de 10-17 mm de longitud, espinas de 1-3 mm de longitud, rectas, marrones o moradas ....................... 10. S. parvifructa Steyerm.

13. Filamentos estaminales esparcidamente hirsutos; cápsula con espinas de 6-9 mm de longitud ......... 6. S. guianensis (Aubl.) Benth.

13. Filamentos estaminales glabros; cápsulas con espinas de 3-5,5 mm de longitud .................................. 8. S. meianthera Donn. Sm.

Sloanea helianthoides Fern. Alonso \& Pal.-Duque, sp. nov. (Figs. 1, 2)

Tipo: Colombia. Cundinamarca: Municipio de Tena, vereda "El Rosario", Orilla de la Laguna Pedro Palo, en potrero, 2100 m. 20-VIII-1984 (fl), E. Barrera 233 (holótipo, COL 321693).

Species typica sectionis Brevispicae Earle Smith; S. brevispina Earle Smith affinis, sed differt inflorescentiis grandioribus usque ad $7 \mathrm{~cm}$ longis, ovario ovoideo vel oblongo, 3,5-5 mm longo, dense pubescente; stylo $3 \mathrm{~mm}$ longo, contorto, ad apicem tribus vel quatuor partibus diviso; capsulis ovoideis vel rotundatis c. $6 \mathrm{~cm}$ longis, $4-5 \mathrm{~cm}$ diametro, 4-5-valvatis, valvis 1,2-1,5 cm crassis, spinis 5-9 mm longis, seminibus plus quam 20,3-4 mm longis, rotundatis vel complanatis.

Árbol de 6-15 m de altura. Corteza café; ramas maduras y jóvenes cubiertas con abundantes lenticelas blancas, de 1-2 mm de longitud, que al unirse dan la apariencia de lenticelas de mayor tamaño (de hasta $8 \mathrm{~mm}$ de longitud). Ramitas maduras gruesas, de $1,2 \mathrm{~cm}$ de diámetro, con la corteza desprendiéndose en pequeñas láminas, glabras o diminutamente pubérulas (indumento amarillento); ramitas jóvenes delgadas, de 4-7 $\mathrm{mm}$ de diámetro, recubiertas con pubescencia amarilla, densa; yemas apicales con indumento denso estrigoso de color blanquecino a amarillento. Hojas alternas; estípulas prontamente caducas; pecíolos de (23)57-90(100) mm de longitud, de 2,8-4 mm de diámetro, redondeados, densamente pubescentes los más jóvenes, los maduros pubérulos a glabrescentes; con dos pulvínulos siendo más conspicuo el distal que el proximal, pulvínulo proximal de $15 \mathrm{~mm}$ de longitud, $5 \mathrm{~mm}$ de diámetro, del mismo color que el pecíolo en las hojas jóvenes o más oscuro en las desarrolladas; láminas desarrolladas de 31,4-36,4 $\mathrm{cm}$ de longitud, 15-22, $8 \mathrm{~cm}$ de ancho, coriáceas, obovadas o menos frecuentemente oblanceoladas; base redondeada o a veces cuneada; ápice redondeado; margen repando; nervio medio parcialmente resaltado y densamente pubescente en la cara abaxial; prominente y esparcidamente pubescente en la cara adaxial; nervios secundarios 12-13 pares, sin llegar a tomar notoriamente una coloración diferente a la de la lámina, levemente excavados y pubérulos en la cara adaxial, prominentes y pubérulos en la cara abaxial; nervios terciarios paralelos y perpendiculares a los secundarios, densamente pubescentes; nervios cuaternarios poligonales, reticulados, pubescentes. Inflorescencias axilares, racemosas; racimos básicos de 6-7 flores; eje primario de $(5,3) 6$ $(7,5) \mathrm{cm}$ de longitud, 1,5-2,5 mm de diámetro, anguloso, recubierto con una densa pubescencia amarillenta; pedicelos de $(0,9) 1,5-2,4(3) \mathrm{cm}$ de longitud y 1-1,5 mm de diámetro, 


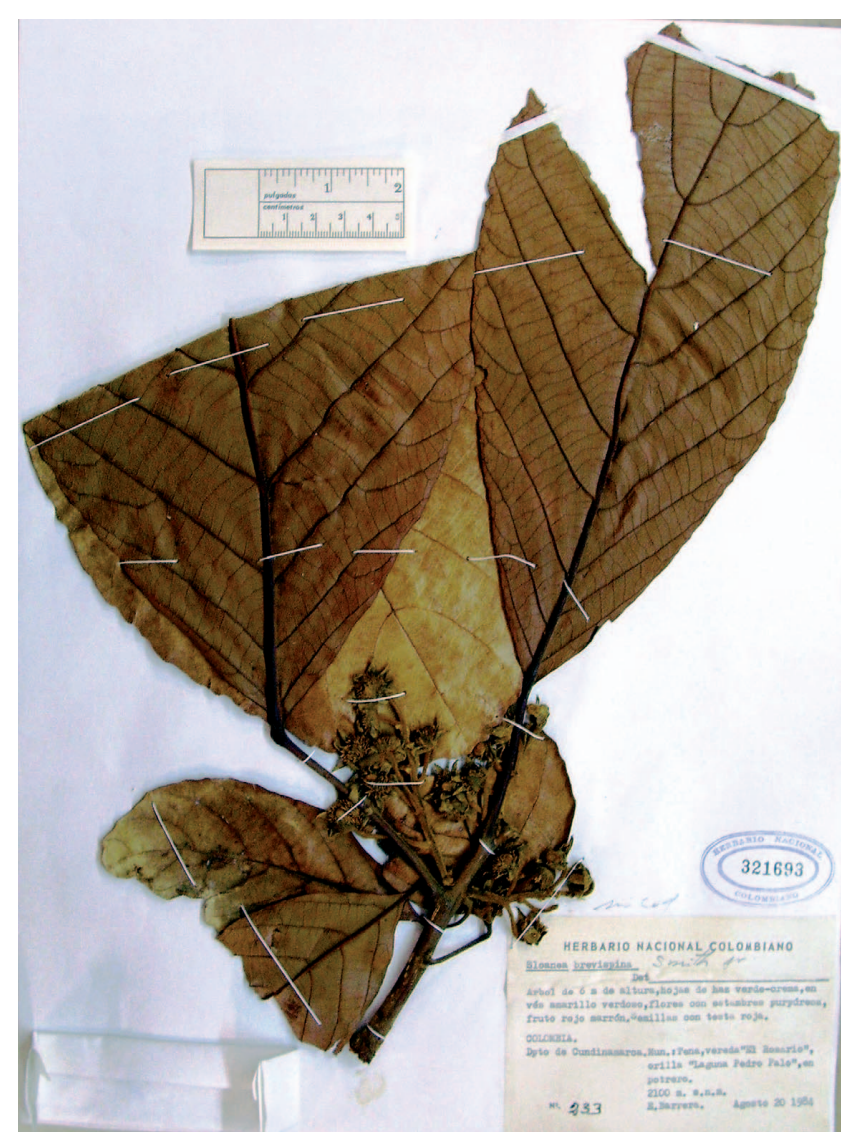

Fig. 1. Holótipo de Sloanea helianthoides, mostrando las inflorescencias. E. Barrera 233 (COL 321693).

angulosos, densamente pubescentes; bractéolas no vistas. Flores con 7-12 sépalos, estrecha a anchamente triangulares, 7-9 mm de longitud, (2)3-4(5) $\mathrm{mm}$ de ancho en la base, densamente pubescentes tanto en la cara adaxial como la abaxial. Estambres de $(3,2) 3,5-4,2(4,5) \mathrm{mm}$ de longitud, de color púrpura (en vivo); filamentos (1,2)1,5-1,8(2) mm de longitud, pubescentes, ligeramente más oscuros (negruzcos) que las anteras; anteras de 1-2 mm de longitud, elípticas (raramente \pm lineares), pubescentes, dehiscentes por dos líneas laterales; conectivo muy tenue, prolongándose hasta un poco más de la mitad de la longitud de la antera sin llegar a la base de la arista; aristas de $0,5 \mathrm{~mm}$ de longitud, pubescentes; receptáculo finamente pubescente, más ancho que el ápice del pedicelo. Ovario ovoide a oblongo, de 3,5-5 $\mathrm{mm}$ de longitud, densamente pubescente; estilo de $3 \mathrm{~mm}$ de longitud, glabro, contorto, dividido en tres a cuatro partes hacia el ápice, que tienden a separarse completamente al final de la antesis. Frutos ovoides a \pm redondeados, c. $6 \mathrm{~cm}$ de longitud y $4-5 \mathrm{~cm}$ de diámetro (calculado a partir de una cápsula abierta); valvas 4-5, leñosas, de 1,2-1,5 cm de grosor; espinas monomórficas, de 5-9 mm de longitud, adelgazándose hacia el ápice, moradas y recubiertas con una abundante pubescencia antrorsa y amarillenta, por lo que toman un color café más o menos cobrizo. Semillas numerosas (más de 20), de 3-4 mm de longitud, redondeadas y algunas veces aplanadas, recubiertas con un arilo más o menos rojizo.

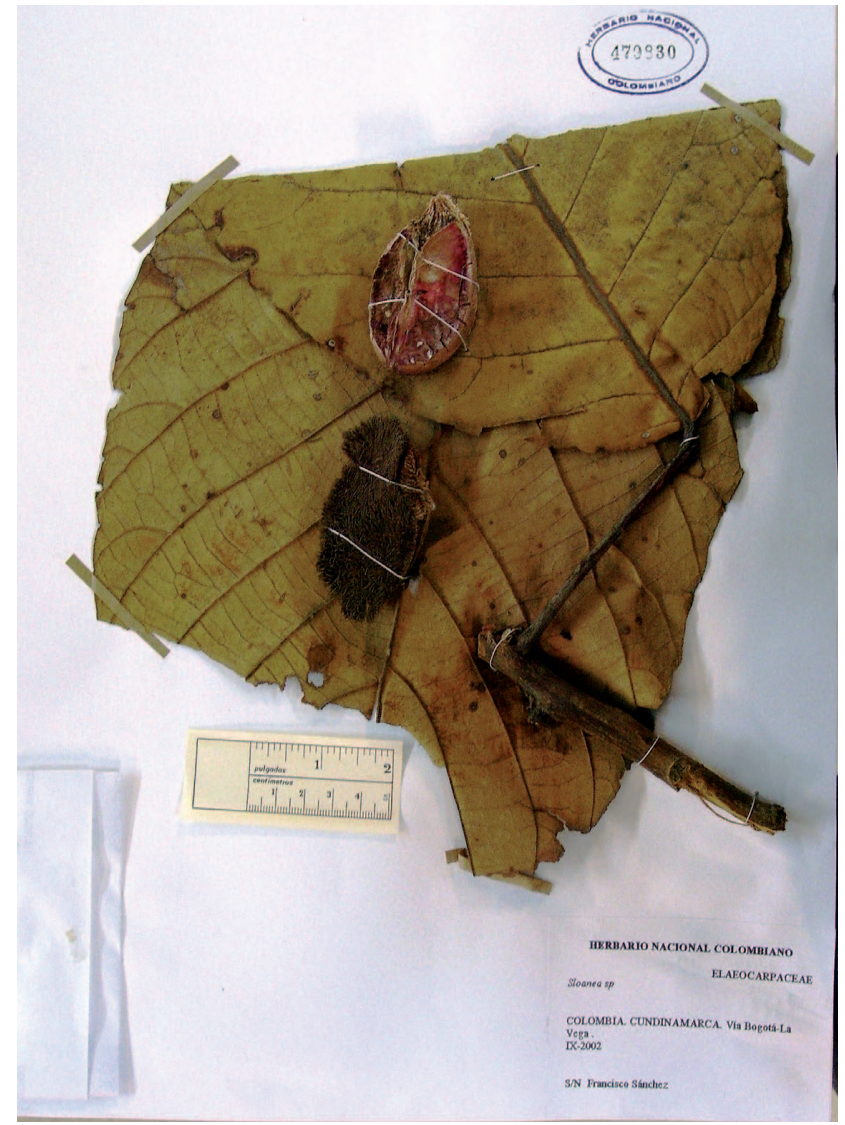

Fig. 2. Uno de los parátipos de Sloanea helianthoides, mostrando una cápsula abierta y parte de sus semillas. F. Sánchez s.n. (COL 479818).

Etimología. El epíteto específico de esta especie hace referencia al aspecto radiado de las flores abiertas, que recuerdan a las representaciones heráldicas del sol y también a los capítulos de Helianthus L. (Asteraceae).

Habitat, distribución y estado de conservación. S. helianthoides por el momento se conoce únicamente por las dos colecciones aquí citadas, efectuadas a $2100 \mathrm{~m}$, ubicadas en la franja de bosque subandino del departamento de Cundinamarca. Ambas corresponden a la cuenca del río Magdalena, en la vertiente occidental de la Cordillera Oriental de Colombia. Esta franja de vegetación está dominada por bosques de robles (Quercus bumboldtii Bonpl.) muy alterados en la actualidad (Lozano \& Torres, 1974; Rangel \& Avella, 2011). Por la escasa información fenológica que se conoce, se sabe que presenta floración en agosto y frutos en septiembre. En cuanto a su estado de conservación, esta especie califica en la categoría de amenaza en peligro crítico (CR), pues solamente se conoce de una localidad, y su área de presencia estimada es menor de $100 \mathrm{~km}^{2}$ (IUCN, 2003). Por consiguiente, se recomienda realizar un diagnóstico de sus poblaciones naturales, en el área en que ha sido herborizada.

Observaciones taxonómicas. S. helianthoides por la coloración café-amarillenta que toman sus hojas cuando secan, por la tonalidad café oscuro de las nervaduras secundarias en el envés y las cápsulas armadas con espinas cortas, se 
podría relacionar con Sloanea brevispina Earle Smith. No obstante, ambas especies se separan claramente por presen$\operatorname{tar} S$. helianthoides hojas obovadas o raramente oblanceoladas, mientras que en $S$. brevispina éstas son ovadas o elíptico-ovadas; las inflorescencias en S. brevispina son de 2-4,5 $\mathrm{cm}$ de longitud, mientras que en $S$. belianthoides son de mayor tamaño, hasta $7 \mathrm{~cm}$ de longitud (Fig. 1). Sus flores también son más grandes y con un mayor número de sépalos 7 12 , los cuales son de mayor tamaño $(7-9 \times(2) 3-4(5) \mathrm{mm}$ de ancho). En S. brevispina las flores tienen c. $4 \mathrm{~mm}$ de longitud y $4 \mathrm{~mm}$ de diámetro y presentan solo 7 sépalos, de c. $1 \mathrm{~mm}$ de longitud. Adicionalmente, los ovarios en S. helianthoides presentan un mayor tamaño y una forma diferente, son de 3,5-5 mm de longitud, oblongo a ovoides y en S. brevispina estos son muy pequeños, de $0,5 \mathrm{~mm}$ de longitud y ovoides. Por último, las cápsulas en $S$. brevispina son más pequeñas, de $3 \times 3 \mathrm{~cm}$ y con una a dos semillas, mientras que en $S$. helianthoides son claramente más grandes, de $6 \times 4-5 \mathrm{~cm}$, con más de 20 semillas por cápsula (Fig. 2) y las espinas de mayor longitud (5-9 $\mathrm{mm}$ ), pues en $S$. brevispina estas últimas sólo tienen como máximo $3 \mathrm{~mm}$ de longitud.

\section{Parátipo}

COLOMBIA. Cundinamarca: Vía Bogotá-La Vega, vertiente occidental de la Cordillera Oriental, IX-2002 (fr), F. Sánchez s.n. (COL 479818; COL 479830).

\section{Sloanea huilaeana Pal.-Duque \& Fern.-Alonso, sp. nov.} (Fig. 3)

Tipo: Colombia. Huila: Vertiente oriental de la Cordillera Central, Mpio. de Santa Leticia, Hacienda Meremberg, km 101 en la vía La Plata-Popayán, 2300 m, 2-XII-1984 (fr), G. Buch s.n. (holótipo, CUVC 11559).

Species typica sectionis Brevispicae Earle Sm.; ex affinitate $S$. granulosae Ducke et $S$. pseudogranulosa Pal.-Duque; a S. granulosa differt capsulis spinis $(0,7) 0,8-1,3(1,4) \mathrm{cm}$ longis (vs. capsulis inermis); a S. pseudogranulosa Pal.-Duque differt capsularum spinis $(0,7) 0,8-1,3(1,4) \mathrm{cm}$ longis, curvatis, non irritantibus (vs. 2-4 mm longis, rectis, irritantibus).

Árbol de $35 \mathrm{~m}$ de altura, por $70 \mathrm{~cm}$ de DAP, con raíces tabulares de 1-1,4 m de altura, delgadas y poco extendidas Tronco recto, cilíndrico; corteza café, fisurada longitudinalmente; ramas maduras de 0,7-1,1 cm de diámetro, café, glabras, fisuradas longitudinalmente y recubiertas con abundantes lenticelas de color blanco; ramas jóvenes de 2-3,5 mm de diámetro, indumento pubérulo de color amarillo, carentes de lenticelas; yemas apicales recubiertas con abundante indumento pubérulo de color amarillo. Hojas alternas, esparcidas sobre las ramas; estípulas no vistas (de $0.5 \mathrm{~mm}$ de ancho en la base, de acuerdo con las cicatrices), prontamente caducas; pecíolos $(0,9) 1,2-1,7(2,1) \mathrm{cm}$ de largo, 1,5-2 mm de diámetro, redondeados, lisos sin ningún tipo de estrías, con abundante indumento pubérulo amarillo; pulvínulos dos, más conspicuo el distal que el proximal; láminas coriáceas, anchamente elípticas a oblongas, de $(9,85) 10-10,6(10,7) \mathrm{cm}$ de largo, $(5,3) 6,5-7,3(7,7) \mathrm{cm}$ de ancho; base obtusa raramente cuneada; ápice obtuso; margen irregularmente ondulado-repando, con diminutos dientes

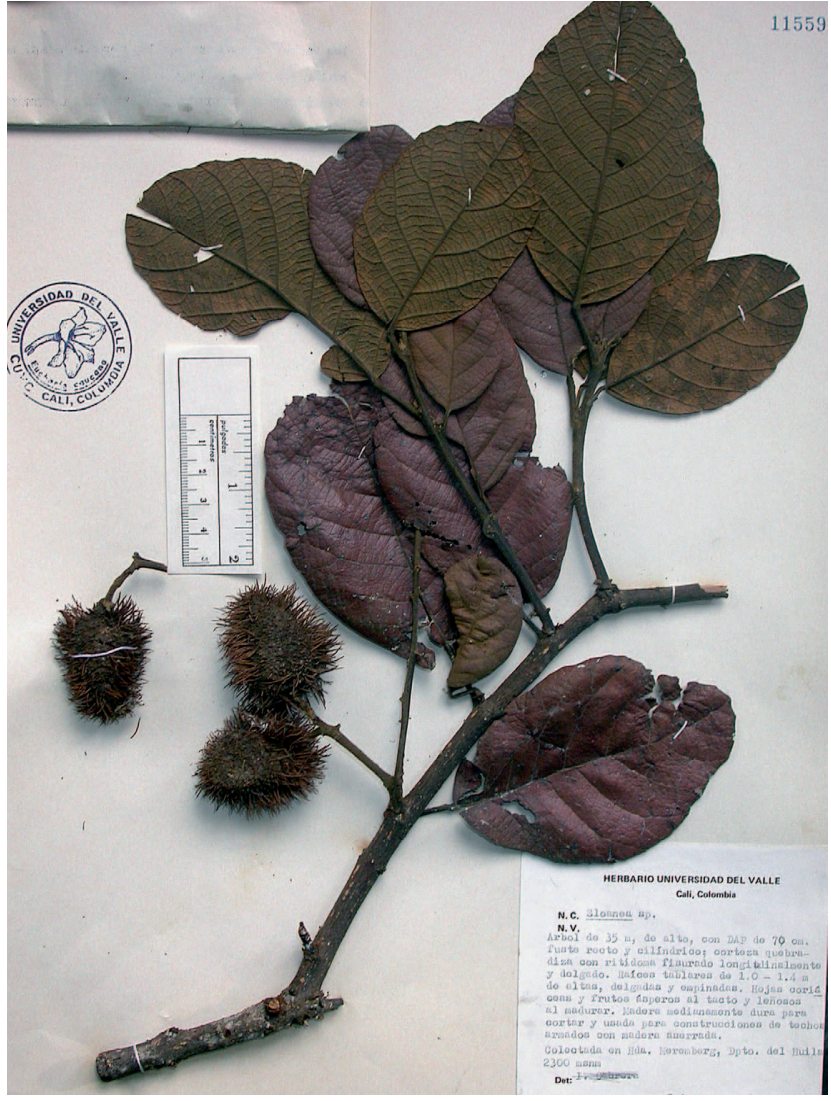

Fig. 3. Holótipo de Sloanea huilaeana, G. Buch, s.n. (CUVC 11559).

que surgen a partir de los nervios secundarios y terciarios; superficie adaxial glabra en hojas maduras y pulverulenta a pubérula en las jóvenes; superficie abaxial completamente recubierta por un indumento pubérulo de color amarillo, densamente dispuesto; venación típicamente craspedódroma simple, nervio principal levemente excavado en la superficie adaxial, prominente en la superficie abaxial, nervios finamente pubérulos; con (7)8-9(10) pares de nervios secundarios, ascendentes en un ángulo de $45^{\circ}-60^{\circ}$, ligeramente excavados y pubérulos en la cara adaxial, glabrescentes en hojas maduras, prominentes y densamente pubérulos en la superficie abaxial; nervios terciarios paralelos entre sí y perpendiculares a los secundarios, levemente excavados y glabros en la cara adaxial, prominentes y finamente pubérulos en la abaxial; nervios cuaternarios poligonales y reticulados, ligeramente cóncavos en la cara adaxial por lo que la lamina toma un aspecto \pm bullado. Inflorescencias no vistas. Flores con sépalos triangulares, 4,5-5,1 $\mathrm{mm}$ de longitud, $3-4 \mathrm{~mm}$ de ancho en la base, finamente pubérulos tanto en la superficie externa como interna. Estambres de $(3,5) 3,7-4,6(5,0) \mathrm{mm}$ de longitud; filamentos de $(1,7) 2-2,5(3) \mathrm{mm}$ de longitud, finamente pubérulos; anteras de (1,5)2-2,1 mm de longitud, lineares, raramente ovadas, finamente pubérulas, dehiscentes por dos líneas laterales, conectivo de $1 \mathrm{~mm}$ de longitud, sin llegar hasta el ápice, del mismo color de la antera, generalmente más conspicuo en la cara abaxial de la antera; aristas ausentes (sépalos y estam- 
bres descritos a partir de unos pocos encontrados en el receptáculo, que persiste en las cápsulas maduras). Infrutescencias racemosas, axilares, ubicadas sobre las ramitas maduras, con al menos tres a cuatro frutos; pedúnculo del racimo de 2,7 $\mathrm{cm}$ de longitud, $3 \mathrm{~mm}$ de diámetro, finamente pubérulo; pedúnculo fructífero de 0,9-1,7 cm de longitud, 2-3 mm de diámetro, finamente pubérulo. Cápsulas ovadas, $3,2-3,5 \mathrm{~cm}$ de longitud ( $\sin$ las espinas), 2-2,3 cm de anchura (sin las espinas); superficie cubierta por un indumento pubérulo no muy conspicuo; espinas densamente dispuestas (a menos de $1 \mathrm{~mm}$ de distancia), de $(0,7) 0,8-1,3(1,4) \mathrm{cm}$ de longitud, persistentes, no irritantes, siendo más largas las de la base y volviéndose más finas hacia el ápice de la cápsula; espinas recubiertas con diminutos tricomas antrorsos; semillas numerosas (15 o más), variables en forma y tamaño, ariladas.

Habitat, distribución y estado de conservación. S. builaeana solamente se conoce por la colección tipo, procedente de la vertiente oriental de la cordillera Central de Colombia, en el departamento del Huila a $2300 \mathrm{~m}$. La zona corresponde a la franja de bosque húmedo montano o bosque de niebla, frecuentemente dominado por el roble Quercus humboldtii Bonpl., que crece con especies como Alchornea grandiflora Müll. Arg. (Euphorbiaceae), Clethra fagifolia Kunth (Clethraceae), Clusia multiflora Kunth (Clusiaceae), Daphnopsis caracasana Meisn. (Thymeleaceae), Hedyosmum racemosum (Ruiz \& Pav.) G. Don (Chloranthaceae) y Rhamnus granulosa (Ruiz \& Pav.) Weberb. Ex M.C. Johnst. (Rhamnaceae). Estos ecosistemas actualmente están fuertemente afectados por cambios en el uso de la tierra para la implementación de cultivos. Aparentemente se trata de una especie muy escasa, pues aunque se han adelantado varios estudios en la región (Rangel \& Franco, 1985; Rangel \& Lozano, 1989; Rangel \& Avella, 2011), solo ha sido recolectada en una ocasión, en 1984. De acuerdo con las categorías de amenaza de la UICN para Colombia, esta especie se incluiría en la categoría En Peligro crítico, (CR) pues tan sólo se conoce por la colección tipo, realizada hace 24 años. Debidoa la intensa destrucción del hábitat natural de la zona, recomendamos realizar un estudio de las posibles poblaciones naturales en la región de procedencia del tipo.

Observaciones taxonómicas. S. builaeana por su venación craspedódroma simple, se relaciona con $S$. granulosa Ducke y $S$. pseudogranulosa Pal.-Duque sección Brevispicae. De la primera especie se separa bien por presentar las hojas esparcidas sobre las ramitas (vs. agrupadas al final de las ramas), laminas anchamente elípticas a más o menos oblongas (vs. elípticas a obovadas), cápsulas armadas con espinas (vs. cápsulas inermes y verruculosas). De la segunda especie se diferencia claramente por las cápsulas armadas con espinas no irritantes de (7)8-13(14) mm de longitud, ligeramente curvas y que se adelgazan hacia el ápice (vs. espinas de 2-4 $\mathrm{mm}$ de longitud, rectas e irritantes). Difiere también notablemente de ambas especies por el abundante indumento pulverulento de color cobrizo en la superficie adaxial, especialmente en las hojas jóvenes.

\section{AGRADECIMIENTOS}

Agradecemos al personal del Instituto de Ciencias Naturales de la Universidad Nacional de Colombia por las facilidades brindadas para el desarrollo de los estudios que actualmente se adelantan en este género. A F. Chiang Cabrera (UNAM), por la corrección de las diagnosis latinas de las especies, e igualmente a los conservadores de los herbarios CAUP, CHOCO, COAH, COL, CUVC, FMB, HUA, HUQ, JAUM, MEDEL, TULV y UDBC, por la colaboración durante las visitas del primer autor o el envío de material en préstamo, especialmente a L.C. Jiménez y O. Rivera (COL), P. Silverstone (CUVC), F.J. Roldán (HUA) y D. Sánchez (MEDEL). A F. Sánchez, del Jardín Botánico José Celestino Mutis de Bogotá, por las colecciones provenientes de San Francisco - La Vega (Cundinamarca). JLFA agradece al CSIC la financiación con cargo al proyecto Intramural CSIC (2009-30I071 y al Ministerio de Ciencia e Innovación de España por el proyecto CGL2010-19747.

\section{REFERENCIAS BIBLIOGRÁFICAS}

Alfaro Castañeda, M.D. 1981. Revisão taxonômica do gênero Sloanea Linnaeus (Elaeocarpaceae) na Amazônia Brasileira. Tesis de grado. Fundação Universidade do Amazonas (FUA), CNPq-INPA, Manaus, Amazonas, Brasil.

Font Quer, P. 1993. Diccionario de Botánica. Ed. Labor, S.A., Barcelona. IUCN. 2001. IUCN Red List Categories and Criteria, Version 3.1. Prepared by the IUCN Species Survival Commission. IUCN, Gland, Switzerland, and Cambridge.

IUCN. 2003. Directrices para emplear los criterios de la Lista Roja de la IUCN a nivel nacional y regional. Versión 3.0. Preparados por la Comisión de supervivencia de Especies de la UICN. IUCN-Unión Mundial para la Naturaleza.

Lozano-C., G. \& Torres, J.H. 1974. Aspectos generales de los bosques de robles (Quercus) en Colombia. Ecología Tropical 1(2): 45-79.

Palacios-Duque, L. 2007. Sloanea esmeraldana Pal.-Duque, sp. nov. (Elaeocarpaceae) del Chocó (Colombia). Anales del Jardín Botánico de Madrid. 64(1): 103-105.

Rangel-Ch, J.O., Franco-R., P. 1985. Observaciones fitoecológicas en varias regiones de vida de la Cordillera Central de Colombia. Caldasia 14: 211-249.

Rangel-Ch, J.O., Lozano-C., G. 1986. Un perfil de vegetación entre La Plata (Huila) y el Volcán del Purace. Caldasia 14:503-547.

Rangel- Ch, J.O. \& Avella, A. (2011): Oak forests of Quercus humboldtii in the Caribbean region and distribution patterns related with environmental factors in Colombia. Plant Biosystems 145: sup1, 186-198.

Sampaio, D. \& Souza, V.C. 2011. Three new species of Sloanea (Elaeocarpaceae). Phytotaxa 16: 45-51.

Smith, C. E. 1954. The new world species of Sloanea (Elaeocarpaceae). Contribution from the Gray Herbarium of Harvard University 175: 1114.

Smith, D. A. 2001. Elaeocarpaceae. In: Stevens, W.D., Ulloa-Ulloa, C., Pool, A. \& Montiel, O.M. (eds.), Flora de Nicaragua. Monographs in Systematic Botany Missouri Botanical Garden 85: 816-820.

Smith, D. A. 2010. Elaeocarpaceae. In: Hammel, B.E., Grayun, M.H., Herrera, C. \& Zamora, N. (eds.), Manual de Plantas de Costa Rica 5. Dicotiledóneas (Clusiaceae-Gunneraceae). Monographs in Systematic Botany Missouri Botanical Garden 119: 222-236.

Smith, D.A. \& Steyermark, J. 1998. Elaeocarpaceae. In: Berry, P.E., Holstm, B.K. \& Yatskievych, K. (eds.), Flora of the venezuelan Guayana. Missouri Botanical Garden Press. 4: 712-729.

Steyermark, J.A. 1988. Flora of the venezuelan Guayana-VI. Annals Missouri Botanical Garden. 75(4): 1565-1586.

Vásquez, R. 2009. Sinopsis del género Sloanea L. (Elaeocarpaceae) en el Perú. Arnaldoa 16(2): 37-63.

Editor asociado: J. Fuertes Aguilar Recibido: 21-XI-2011 Aceptado: 24-V-2012 
